\title{
Fermionic Path Integration and Grassmann Brownian Motion*
}

\author{
Alice Rogers \\ Department of Mathematics, King's College, Strand, London WC2R 2LS, United Kingdom
}

\begin{abstract}
A Grassmann probability theory, with anticommuting random variables and stochastic processes, is developed using an extension of Berezin integration to infinite dimensional spaces. A Kolmogorov-type consistency condition allows integration on spaces of paths in anticommuting space. One particular stochastic process, Grassmann Brownian motion, is described and the associated measure used to give a path-integral formula for the kernel of the evolution operator in fermionic quantum mechanics. The Fourier mode expansion of Grassmann Brownian motion is derived.
\end{abstract}

\section{Introduction}

Path integration techniques are among the most powerful in present-day quantum physics; the quantisation of a new type of theory often proceeds, at least in the first instance, by summing over everything, with more or less plausible weights. While this is a good heuristic procedure, and formal manipulation of path integrals often gives extremely valuable insight, ultimately the true meaning (if any) of the path integrals in a theory should be established. This is particularly true in the case of path integrals for fermions, where the integrals are not even the limits of sums, and standard measure theoretical results do not apply. For instance, it is usually assumed that the sum over paths can be replaced by a sum over fourier modes, which is far from obvious when the word sum is being used in a formal sense. (In fact one result of this paper will be to justify this procedure, and to give the correct normalization.)

The analytic theory of path integration in imaginary time bosonic quantum mechanics is fully understood. The basic approach, (which goes back to ideas of Wiener, Feynman and Kac), together with many applications (and references to original work) is described in the excellent book of Simon [1]. In this paper an analytic theory of fermionic path integration is constructed, in a manner as closely

* Research supported by the Science and Engineering Research Council of Great Britain under advanced research fellowship number $\mathrm{B} / \mathrm{AF} / 687$ 
analogous as possible to the bosonic method. An important difference is that there is no existing integration theory for spaces of anticommuting variables of uncountably infinite dimension which reduces to Berezin integration in the finite-dimensional case; this paper constructs such a theory, in a manner analogous to Bochner's treatment of conventional stochastic processes [2], where the primary objects are the finite dimensional marginal distributions. It is generally the case that fermions only involve finite dimensional algebra; here, because of the presence of the continuous time parameter, some genuine analysis is required.

The approach in this paper is specifically designed to make it possible to extend rigorous path integral techniques to theories involving both bosons and fermions, and in particular to supersymmetric theories [3], and to fermi fields in curved space. Path integrals not only give physical results, they also have mathematical uses; for instance, the very simple proofs of the Atiyah-Singer index theorem using supersymmetric quantum mechanics [4] use fermionic path integration.

In Sect. 2 the representation of fermionic quantum mechanics in terms of functions of anticommuting variables [5] is reviewed, together with general techniques for handling functions of such variables. Differential operators, and their kernels, are defined; the path integral developed in later sections gives a method for evaluating the kernel of the evolution operator of a fermionic system.

Brownian motion in a space of anticommuting variables is described in Sect. 3; in order to do this, an extension of the Berezin integral to infinite dimensional spaces is developed, together with a certain amount of what might be called Grassmann probability theory. The Brownian motion is also expressed as a Fourier sum of independent (Grassmann) Gaussians, thus making rigorous the heuristic physicist's replacement of the sum over paths by the sum over Fourier modes with anticommuting boundary conditions [6].

Section 4 contains a proof of the Feynman-Kac formula for fermions, that is the expression of the kernel of $e^{-H t}$ in terms of a path integral, the measure being that associated with the Brownian motion developed in the proceeding section. The formula is applied to calculate the heat kernel of the fermionic oscillator; as is often the case when actually calculating path integrals, the Fourier mode version is used. One reason for considering the oscillator in detail is that a fermi field can be regarded as an infinite collection of such oscillators. (This approach to fermi fields is described in detail in the book of Fadeev and Slavnov [7].)

There is a considerable literature on various non-commutative versions of probability theory $[8,9]$; however these theories do not seem to be directly applicable to the type of path integrals considered in this paper. The closest is the work of Haba [9] but the anticommuting Brownian motion constructed there is in position space, not phase space, and would not lead to the Feynman-Kac formula presented here.

\section{Differential Operators in Anticommuting Space, and the Grassmann Formulation of Fermionic Quantum Mechanics}

In this section a representation of the canonical anticommutation relations of fermionic quantum mechanics is constructed using differential operators on a space 
of anticommuting Grassmann variables. The necessary techniques of "analysis" of functions of Grassmann variables are described.

In the canonical quantisation of $n$-dimensional particle mechanics, the classical observables $p^{i}$ (momentum) and $x^{i}$ (position) $(i=1, \ldots, n)$ are replaced by the quantum operators $\hat{p}^{i}$ and $\hat{x}^{i}$, also the classical Poisson brackets

$$
\left\{x^{i}, p^{j}\right\}=\delta^{i j}
$$

are replaced by the quantum commutation relations

$$
\left\{\hat{x}^{i}, \hat{p}^{j}\right\}=i h \delta^{i j} .
$$

The standard representation is the Schrödinger representation, where $\hat{p}^{i}$ and $\hat{x}^{i}$ are represented on the space $L^{2}\left(\mathbb{R}^{n}\right)$ of square integrable complex valued functions of $\mathbb{R}^{n}$ by

$$
\begin{aligned}
& \hat{p}^{i} f(x)=-i \partial / \partial x^{i}(f)(x), \\
& \hat{x}^{i} f(x)=x^{i} f(x) .
\end{aligned}
$$

(As usual, units are used in which Planck's constant $h$ is set to one.)

In an analogous fashion systems with spin in $n$ dimensions may be considered by introducing operators $\hat{\psi}^{i}(i=1, \ldots, n)$ which act on the space of polynomial functions of $n$ anticomputing variables.

Following Berezin and Marinov [5], one begins with a "classical" Hamiltonian $H=V(\psi)$ (the $\psi^{i}$ being anticommuting variables) and symmetric Poisson brackets

$$
\left\{\psi^{i}, \psi^{j}\right\}=2 \delta^{i j} \text {. }
$$

On quantisation one replaces the anticommuting variables $\psi^{i}$ by operators $\hat{\psi}^{i}$ which satisfy the anticommutation relations

$$
\left\{\hat{\psi}^{i}, \hat{\psi}^{j}\right\}=2 \delta^{i j} \text {. }
$$

The "Schrödinger" representation is achieved $[7,10]$ by defining states to be members of a carefully defined class of functions of $n$ anticommuting variables $\theta^{i}, \ldots, \theta^{n}$, and setting

$$
\hat{\psi}^{i}=\widehat{\operatorname{def}} \theta^{i}+\partial / \partial \theta^{i}
$$

Finally one considers the Schrödinger equation

$$
\partial f / \partial t=-H(\hat{\psi}) f .
$$

(As with bosonic quantum mechanics, there may be ambiguities in operator orders to be resolved in the transition from the classical $H(\psi)$ to the quantum $H(\hat{\psi})$.) The fermionic system is solved if one can find the kernel of the evolution operator $\exp (-H t)$; the next two sections of this paper are devoted to developing a rigorous path-integral method for obtaining these kernels.

Some details of the analysis of functions of anticommuting variables will now be described. (Many further details may be found in [11].) Concrete rather than abstract anticommuting variables are used because the language involved is much simpler in the case of concrete variables. 
For each positive integer $L$, let $B_{L}$ denote $\Lambda\left(\mathbb{R}^{L}\right)$, the Grassmann algebra over $\mathbb{R}^{L}$ and let $C_{L}$ denote $\Lambda\left(\mathbb{C}^{L}\right)$, the Grassmann algebra over $\mathbb{C}^{L}$. Thus $B_{L}\left(C_{L}\right)$ is the algebra over the real (complex) field with generators $1, b_{1}, \ldots, b_{L}\left(1, c_{1}, \ldots, c_{L}\right)$ and relations

$$
\begin{aligned}
& b_{i} b_{j}=-b_{j} b_{i} \\
& c_{i} c_{j}=-c_{j} c_{i} \quad(i, j=1, \ldots, L)
\end{aligned}
$$

respectively. The unique algebra homomorphism of $B_{L}$ into $C_{L}$ which maps $b_{i}$ onto $c_{i}(i=1, \ldots, L)$ defines an embedding of $B_{L}$ into $C_{L} . B_{L, 0}$ and $B_{L, 1}$ denote the even and odd parts of $B_{L}$ respectively, while $B_{L}^{m, n}$ denotes the cartesian product of $m$ copies of the even part of $B_{L}$ and $n$ copies of the odd part (with similar notation for $C_{L}$ ).

Some useful classes of functions of the space $B_{L}^{0, n}$ of $n$ anticommuting variables will now be defined; basically all functions will be polynomials in the odd variables; such different possibilities as there are come from allowing the coefficients to take values in different spaces.

Definition 2.1. (a) $H^{\infty}\left(B_{L}^{0, n}, B_{L}\right)$ denotes the set of functions

such that

$$
f: B_{L}^{0, n} \rightarrow B_{L}
$$

$$
f\left(\theta^{1}, \ldots, \theta^{n}\right)=f_{\phi}+\sum_{i=1}^{n} f_{i} \theta^{i}+\sum_{i<j=1}^{n} f_{i j} \theta^{i} \theta^{j}+\cdots=\sum_{\mu \in M_{n}} f_{\mu} \theta^{\mu}
$$

(Here $\mu$ is a multi index and $M_{n}$ is the set of all multi indices $\mu_{1} \ldots \mu_{k}$ $1<\mu_{1}<\cdots<\mu_{k}<n$, together with the "empty" index denoted $\phi$, and each $f_{\mu}\left(\mu \in M_{n}\right)$ is a real number.)

(b) Using the embedding of $B_{L}$ into $C_{L}, H^{\infty}(n)$ is defined to be the set of functions of $B_{L}^{0, n}$ into $C_{L}$ of the form (2.11) with the coefficients $f_{\mu}$ now allowed to be complex.

(c) $G H^{\infty}\left(B_{L}^{0, n}, B_{L}\right)$ is the set of functions of the form (2.11) with the coefficients $f_{\mu}$ taking values in $B_{L / 2}$. (Here and elsewhere, the natural embedding of $B_{L / 2}$ in $B_{L}$, and of $C_{L / 2}$ in $C_{L}$, will be implicitly used.)

(d) $G H^{\infty}(n)$ is the set of functions of the form (2.11) with the coefficients $f_{\mu}$ taking values in $C_{L / 2}$.

Differentiation with respect to $\theta^{i}$ takes the natural form

$$
\begin{aligned}
\partial / \partial \theta^{i}\left(f_{\mu} \theta_{\mu}\right) & =-(-1)^{r+\left|f_{\mu}\right|} f_{\mu} 1 \theta^{\mu_{1}} \ldots \hat{\theta}^{\mu_{r}} \ldots \theta^{\mu_{k}} \quad \text { if } \quad i=\mu_{r} \\
& =0 \text { otherwise, }
\end{aligned}
$$

extending linearly to all $H^{\infty}$ and $G H^{\infty}$ functions. (Here $\left|f_{\mu}\right|$ denotes the Grassmann parity of $f_{\mu}$.)

In order to avoid unwanted cancellations, it is necessary to use a value of $L$ (the number of anticommuting generators) greater than $2 n$. Often in this paper one has to use a space $B_{L}^{(0, n) N}$ consisting of $N$ copies of the space $B_{L}^{0, n}$; or even the space $B_{L}^{(0, n) A}$ consisting of copies of $B_{L}^{0, n}$ indexed by an arbitrary set $A$. In these cases, again in order to avoid unwanted cancellations, rather than using true copies 
one must use a different set of generators for each factor; thus strictly

$$
B_{L}^{(0, n) N}={ }_{1} B_{L}^{0, n} \times \cdots \times{ }_{N} B_{L}^{0, n},
$$

where ${ }_{r} B_{L}(r=1, \ldots, N)$ is the Grassmann algebra with $L$ anticommuting generators ${ }_{r} b_{1}, \ldots,{ }_{r} b_{L} . B_{L}^{(0, n) A}$ is defined similarly.

Also if $B$ is a finite subset of $A$,

$$
{ }_{B} B_{L}={ }_{1} B_{L} \times \cdots \times{ }_{k} B_{L}
$$

with ${ }_{B} C_{L}$ defined similarly.

$H^{\infty}(n B)$ is the set of functions of $B_{L}^{(0, n) B}$ into ${ }_{B} C^{L}$ of the form

$$
f\left(\theta^{i a}\right)=\sum_{\mu \in M_{n|B|}} f_{\mu} \theta^{\mu}
$$

with each $f_{\mu}$ complex. $G H^{\infty}(n B)$ is the set of functions of the form (2.15) with the coefficients $f_{\mu}$ taking values in $C_{L / 2}$.

The norm used on each Grassmann algebra is the $1^{1}$ norm, since with this norm the Grassmann algebras are Banach algebras.

A representation of the canonical anticommutation relations (2.6) on $H^{\infty}(n)$ (or on any of the other classes of functions of $B_{L}^{0, n}$ which are defined above) is obtained if one sets

$$
\hat{\psi}^{i}=\theta^{i}+\partial / \partial \theta^{i} \quad(i=1, \ldots, n) .
$$

In order to include time evolution one must in fact consider an extended set of functions with the coefficients of the polynomials in $\theta$ being suitably smooth functions of the real variable time. The Schrödinger equation (2.8) then has meaning, and one last technical preliminary remains, the definition of the integration measure which will be used to define the kernel of the evolution operator.

For integration on $B_{L}^{0, n}$ one uses the familiar Berezin rule [12]

$$
\int d^{n} \theta f(\theta)=f_{1 \ldots n},
$$

if $f(\theta)=f_{1 \ldots n} \theta_{1} \ldots \theta_{n}+$ lower order terms. (In the next section this method of integration will be extended to finite dimensional spaces.)

It is now possible to define the kernel of an operator on $H^{\infty}(n)$; the Feynman-Kac formula (Theorem 4.2) will express the kernel of $e^{-H t}$ as a path integral.

Definition 2.2. Given an operator $K$ on the space $H^{\infty}(n)$, if there exists a function $[K]$ in $H(2 n)$ such that

$$
K f(\theta)=\int d^{n} \phi[K](\theta, \phi) f(\phi),
$$

then the function $[K]$ is called the kernel of the operator $K$. An important case is the delta function, that is, the kernel of the identity operator $I$. As usual one has

$$
[I]=\widehat{\operatorname{def}} \delta(\theta, \phi)=\prod_{i=1}^{n}\left(\theta^{i}-\phi^{i}\right)
$$

which satisfies

$$
f(\theta)=\int d^{n} \phi \delta(\theta, \phi) f(\phi) .
$$


For the derivation of the Feynmann-Kac formula in Sect. 4 it is useful to use the Fourier-transform version

$$
\delta(\theta, \phi)=\int d^{n} \rho \exp i(-\rho \cdot(\theta-\phi)),
$$

where

$$
\rho \cdot(\theta-\phi)=\widehat{\operatorname{def}} \sum_{i=1}^{n} \rho^{i}\left(\theta^{i}-\phi^{i}\right)
$$

Also, since the delta function is an $H^{\infty}$ function, it can be shown that for any differential operator $K$ acting on $H^{\infty}(n)$, one has

$$
[K](\theta, \phi)=K_{\theta}(\delta)(\theta, \phi),
$$

where the subscript $\theta$ indicates that derivatives are being taken with respect to the $\theta$ variable.

A useful application of this result is that

$$
\left[\hat{\psi}^{\mu}\right](\theta, \phi)=\int d^{n} \rho(\theta+i \rho)^{\mu} \exp i(-\rho .(\theta-\phi)),
$$

where, if the multiindex $\mu=\mu_{1} \ldots \mu_{k}$,

then

$$
\widehat{\psi}^{\mu}=\widehat{\operatorname{def}} \widehat{\psi}^{\mu_{1}} \ldots \widehat{\psi}^{\mu_{k}}
$$

and so on.

So far a representation of the canonical anticommutation relations (2.6), that is

$$
\left\{\hat{\psi}^{i}, \hat{\psi}^{j}\right\}=2 \delta^{i j}
$$

has been defined on the $2^{n}$ dimensional complex vector space $H^{\infty}(n)$. Obviously the anticommutation relations are simply the defining relations of the $n$ dimensional Clifford algebra, and for some applications it will be useful to construct the $2^{n / 2}$ dimensional representations. This may be achieved by restricting to an appropriate subspace of $H^{\infty}(n)$; the details are not relevant to the rest of this paper. Alternatively one may represent the operators $\hat{\psi}^{i}$ directly on the space of $H$ functions of $n / 2$ complex anticommuting Grassmann variables $a^{j}$ by

$$
\begin{aligned}
\hat{\psi}^{2 j-1} & =a^{j}+\partial / \partial a^{j} \\
\hat{\psi}^{2 j} & =i\left(a^{j}-\partial / \partial a^{j}\right) \quad j=1, \ldots, n / 2,
\end{aligned}
$$

and modify the measure defined in Sect. 3 accordingly.

Physically meaningful answers are always of course real numbers, not Grassmann numbers; these are achieved by taking traces. It is easily shown that, for a linear operator $K$ (acting on $H^{\infty}(n)$ regarded as a $2^{n}$ dimensional complex vector space) with kernel $[K]$ one has

$$
\operatorname{trace} K=\int d^{n} a[K](a,-a) .
$$

(The minus sign in front of the second a simply compensates for sign changes which occur when there are an odd number of anticommuting factors.) 


\section{Anticommuting Brownian Motion}

In order to construct a Feynman-Kac formula for fermions, a measure (in the sense of some infinite extension of Berezin integration) on the space of paths in anticommuting space $B_{L}^{0, n}$ is required. In this section a general formalism for these measures will be developed, along with concepts such as the Grassmann random variable and the Grassmann stochastic process. One particular process, Grassmann Brownian motion, and the associated measure, Grassmann Wiener measure, will be constructed. This measure will be used in the Feynman-Kac formula for fermions established in the next section. The Fourier-mode version of the process will also be constructed; this is useful when actually evaluating path integrals. First some informal comments which motivate the definition will be given.

In bosonic quantum mechanics the physicist's notation for the matrix elements of states evolving according to the Hamiltonian $H=\hat{p}^{2}+V(\hat{x})$ is

$$
\left\langle q\left|e^{-H t}\right| q^{\prime}\right\rangle=\int D x \exp \left(-\int_{0}^{t} \dot{x}^{2}+V(x) d t\right),
$$

where the integration is over all paths beginning at $q$ and ending at $q^{\prime}$. The corresponding analytic formula is [1]

$$
e^{-H t}\left(q, q^{\prime}\right)=\int d \mu\left(q, q^{\prime}\right) \exp \left(-\int_{0}^{t} V(x) d t\right),
$$

where $\mu$ is the conditional Wiener measure on the space of paths in $\mathbb{R}$. By including the kinetic term $\dot{x}^{2}$ in the measure rather than in the integrand a true measure, free from infinite dimensional normalisation factors and so on, is obtained. Using the notation $\{b(t)\} t>0$ for Brownian motion, the joint distribution of $\left(b\left(t_{1}\right)\right.$, $\left.b\left(t_{2}\right), \ldots, b\left(t_{n}\right)\right) 0<t_{1}<\cdots<t_{n}$ is

$$
P_{s}\left(0, x_{1}\right) P_{t}\left(x_{1}, x_{2}\right) \ldots P_{t}\left(x_{n-1}, x_{n}\right) d^{n} x
$$

where $s_{1}=t_{1}, s_{2}=t_{2}-t_{1}, \ldots, s_{n}=t_{n}-t_{n-1}$ and

$$
P_{t}(x, y)=(2 \pi t)^{-1 / 2} \exp \left(-(x-y)^{2} / 2 t\right) .
$$

This corresponds to the kinetic term contribution in the physicist's more heuristic approach. $P_{t}(x, y)$ is simply the kernel of the operator $\exp \left(-t \hat{p}^{2}\right), \hat{p}^{2}$ being the free Hamiltonian.

In the fermionic case, where the free Hamiltonian is zero, one might expect to use the delta function (2.19) in place of $P_{t}(x, y)$. In fact to derive the Feynman-Kac formula one has to use the phase space or Fourier transform expression (2.21) for the delta function, and define a measure on the space of paths in $B_{L}^{0,2 n}$. Thus one is led to consider the $B_{L}^{0,2 n}$ valued process $\left\{\left(\theta^{i}(t), \rho^{1}(t)\right)\right\}(i=1, \ldots, n) t>0$ with finite dimensional joint distributions having the form

$d^{n N} \theta d^{n N} \rho \exp i\left(-\rho_{1} \cdot\left(\theta_{1}-b\right)-\rho_{2} \cdot\left(\theta_{2}-\theta_{1}\right)-\cdots-\rho_{N} \cdot\left(\theta_{N}-\theta_{N-1}\right)-k \cdot\left(a-\theta_{N}\right)\right)$,

$a, b$ and $k$ being three anticommuting parameters. 
At this stage this is simply a heuristic picture. The theory of Grassmann probability spaces will now be developed to give these expressions meaning. First one must define an extension of Berezin integration to functions on $B_{L}^{(0, n) A}$, where $A$ is an arbitrary topological space. (Generally $A$ will be an interval of the real line.) The literature contains various approaches to Berezin integration on countably infinite dimensional spaces [13], mostly in the context of fermionic quantum field theory, and also the earlier work of Segal on anticommuting integration and non-commutative $L^{p}$ spaces [14]. The approach taken in this paper is somewhat different, being constructed with path integration specifically in mind.

Definition 3.1. An $n$-Grassmann probability space of weight $w \in C_{L}$ consists of

(a) a set $A$;

(b) the space $B_{L}^{(0, n) A}$;

(c) for each finite subset $B$ of $A$ a function $f_{B} \in G H^{\infty}(n|B|)$ such that

(i) $\int d^{n|B|} \theta f_{B}(\theta)=w$.

(ii) if $B=\left\{b_{1}, \ldots, b_{s}\right\}$ and $B^{\prime}=\left\{b_{1}, \ldots, b_{s-1}\right\}$, then

$$
f_{B^{\prime}}\left(\theta^{i 1}, \ldots, \theta^{i s-1}\right)=\int d^{n} \theta^{s} f_{B}\left(\theta^{i 1}, \ldots, \theta^{i s}\right) .
$$

Such a space will be denoted $\left(B_{L}^{(0, n) A},\left\{f_{B}\right\}, d \mu\right)$.

The idea in this approach has been to build Kolmogorov-type consistency conditions into the definition and thus be able to integrate certain objects, not quite functions on $B_{L}^{(0, n) A}$, which will be called Grassmann random variables.

Definition 3.2. (a) A Grassmann random variable $g$ on a Grassmann probability space $\left(B_{L}^{(0, n) A},\left\{f_{B}\right\}, d \mu\right)$ consists of

(i) a set $C \subset A$.

(ii) a sequence $B_{1}, B_{2}, \ldots$ of finite subsets of $C$ such that $\bigcup_{r=1} B_{r}$ is dense in $C$.

(iii) a sequence of functions $g_{1}, g_{2}, \ldots$ with $g_{r} \in G H^{\infty}\left(n B_{r}\right)$ for each $r=1,2, \ldots$ such that $\int d^{n|B|} \theta f_{B}(\theta) g_{r}(\theta)$ tends to a limit as $r$ tends to infinity. This limit will be denoted $\int d \mu g$ and called the expectation of $g$.

(b) A Grassmann random variable is said to be odd if each function $g_{r}$ takes values in the odd part of ${ }_{B} B_{L}$.

(c) Let $\left\{g^{k}, k=1, \ldots, N\right\}$ be a set of $N$ odd Grassmann random variables based on the same set $C \subset A$, and the same sequence $B_{1}, B_{2}, \ldots$ of finite subsets of $C$. Also, for each $k=1, \ldots, N$ let $g_{r}^{k}(r=1,2, \ldots)$ be the sequence of functions which defines the random variable $g^{k}$. Then if, for each $v$ in $M_{N}$, the sequence $g_{r}^{v}$ defines a Grassmann random variable, the set $\left\{g^{k}, k=1, \ldots, N\right\}$ is called an $N$-dimensional Grassmann random variable.

(d) Let I be an interval of the real line. A collection $\left\{w^{k}(t) \mid k=1, \ldots, N, t \in I\right\}$ of $N$ dimensional Grassmann random variables indexed by $I$ is called an $N$-dimensional stochastic process if each finite subset is a Grassmann random variable of the appropriate dimension.

(e) If $\left\{g^{k} \mid k=1, \ldots, N\right\}$ is an $N$-dimensional Grassmann random variable, then the function $h$ in $G H^{\infty}(N)$ such that for each $v$ in $M_{N}$

$$
\int d \mu g^{v}=\int d^{N} \theta h(\theta) \theta^{v}
$$


is called the joint probability distribution of $\left\{g^{1}, \ldots, g^{N}\right\}$.

(f) Two $N$-dimensional Grassmann stochastic processes

$$
\left\{w^{k}(t) \mid t \in I\right\} \quad \text { and } \quad\left\{w^{\prime k}(t) \mid t \in I\right\}
$$

are said to be versions of each other if the joint distributions of

$$
\left\{w^{k}\left(t_{1}\right), \ldots, w^{k}\left(t_{N}\right)\right\}
$$

and

$$
\left\{w^{\prime k}\left(t_{1}\right), \ldots, w^{\prime k}\left(t_{N}\right)\right\}
$$

are the same for each finite subset $\left\{t_{1}, \ldots, t_{N}\right\}$ of $I$.

At this stage it would be possible to develop a theory of Grassmann probability spaces with many concepts and results analogous to those of Bochner's approach to stochastic processes [2]. Here only those aspects relevant to fermionic path integration will be developed. One concept which will be necessary is the concept of independent random variables. Two Grassmann random variables will be said to be independent if their joint distribution $f\left(\theta^{r}, \phi^{s}\right)$ factorises as $g\left(\theta^{r}\right) h\left(\phi^{s}\right)$. If they are of the same dimension it can be shown that the distribution of their sum is

$$
f^{\prime}\left(\theta^{i}\right)=\int d^{n} \phi g(\theta-\phi) h(\phi)=\int d^{n} \phi g(\phi) h(\theta-\phi) .
$$

Independence of a finite set of Grassmann random variables is defined similarly.

It will also be useful to have a concept of Gaussian Grassmann random variables.

Definition 3.3. (a) An $N$-dimensional Grassmann random variable $\left(\theta^{r}\right) r=1, \ldots, N$ is said to be Gaussian if its joint probability distribution is of the form

$$
\int d^{n} \rho \exp \left(-i \sum_{r=1}^{N}\left(\rho^{r} \theta^{r}\right)-\sum_{r, s=1}^{N}\left(C^{r s} \rho^{r} \rho^{s}\right)\right),
$$

where the $C^{r s}$ are real numbers and

$$
C^{r s}=-C^{r s}
$$

(b) A Grassmann stochastic process is said to be Gaussian if all finite subsets are Gaussian.

Part (a) of this definition allows a delta function to be a degenerate Gaussian with $C^{r s}=0$. It may easily be shown that $C^{r s}$ is the covariance of $\left(\theta^{r}, \theta^{s}\right)$, (that is, the expectation of $\theta^{r} \theta^{s}$,) and consequently the covariances of a finite set of jointly Gaussian random variables determines their joint distribution. For a Gaussian stochastic process one has the following result.

Proposition 3.4. Let $A$ be an interval in $\mathbb{R}$ and $C: A \times A \rightarrow \mathbb{R}^{n}$

$$
(s, t) \mapsto\left(C^{i j}(s, t)\right),
$$

with

$$
C^{i j}(s, t)=-C^{j i}(t, s)
$$

Then there exists a jointly Gaussian $n$ dimensional Grassmann stochastic process 
$\left\{w^{i}(t) \mid t \in A\right\}$ such that

$$
E\left(w^{i}(s) w^{j}(t)\right)=C^{i j}(s, t)
$$

for all $i, j=1, \ldots, n$ and all $s, t$ in $A$. Also, if $\left\{w^{\prime}(t) \mid t \in A\right\}$ is another $n$-Grassmann stochastic process satisfying these conditions, then the two processes are versions of each other. Outline of proof: $\left(B_{L}^{(0, n) A},\left\{f_{B}\right\}, d \mu\right)$ with, given $B=\left\{t_{1}, \ldots, t_{n}\right\} \subset A$,

$$
f_{B}=\operatorname{def} \int d^{N n} \rho \exp \left(-i \sum_{r=1}^{N} \rho^{r} \cdot \theta^{r}+\sum_{r, s=1}^{N} \sum_{i, j=1}^{n} C^{i j}\left(t_{r}, t_{s}\right) \rho^{i r} \rho^{j} S\right)
$$

is an $n$-Grassmann probability space. Also $\left\{\theta^{i}(t) \mid t \in A\right\}$ is then a Gaussian Grassmann stochastic process with the correct distribution.

Another useful property of Gaussian Grassmann random variables is that the sum of two independent $N$ dimensional Gaussian Grassmann random variables with covariances $C$ and $C^{\prime}$ is an $N$-dimensional Gaussian random variable with covariance $C+C^{\prime}$.

The Grassmann probability space used in fermionic path integration can now be defined, using the formal concepts just developed to make sense of the distribution (3.5).

Definition 3.5. (a) Let $A$ be the interval $(0, \infty)$ and $a, b$ and $k$ be odd elements in $B_{L}$. Then $n$-Grassmann Wiener space with parameters $a, b, k$ is the $2 n$-Grassmann probability space $\left(B_{L}^{(0,2 n) A},\left\{f_{B}, d \mu\right\}\right)$ with, given $B=\left\{t_{1}, \ldots, t_{N}\right\} \subset A$,

$$
f_{B}=\exp i\left(-\rho^{1} \cdot\left(\theta^{1}-b\right)-\rho^{2} \cdot\left(\theta^{2}-\theta^{1}\right) \ldots-\rho^{N} \cdot\left(\theta^{N}-\theta^{N-1}\right)-k \cdot\left(a-\theta^{N}\right)\right)
$$

(b) The corresponding $2 n$-Grassmann probability measure will be called $n$-Grassmann Wiener measure between $a$ and $b$ with momentum $k$, and denote $d \mu[a, b, k]$.

(c) The $n$-dimensional Grassmann stochastic process $w(t)[a, b, k]$ with $w(t)=$ $\theta(t)+i \rho(t)$ will be called $n$-Grassmann Brownian motion between $a$ and $b$ with momentum $k$. (It is straightforward to check that the functions $f_{B}$ of Eq. (3.15) do satisfy the consistency conditions (3.7).)

The extra parameter $k$ (in addition to the parameters $a$ and $b$ which one might expect by analogy with conventional conditional Wiener measure) arises because one is working in phase space. It is possible to calculate the $N$-point distributions of Grassmann Brownian motion, and use them instead of (3.15), thus using an $n$-Grassmann rather than a $2 n$-Grassmann probability space; for the results needed in this paper (3.15) is generally simpler to use, and essential to the proof of the fermionic Feynman-Kac formula. In other contexts, such as developing a lattice approach to fermionic quantum field theory, the $n$-dimensional space might be more useful.

A result concerning integration with respect to Grassmann Wiener measure will now be proved. This result is crucial to the Feynman-Kac formula which will be constructed in the next section.

Proposition 3.6. Let $f_{1}, \ldots, f_{N} \in H^{\infty}(n)$ and $t_{1}, \ldots, t_{N}$ be real numbers with $0<t_{1}<\cdots<t_{N}$. Then

$$
\left[f_{N}(\hat{\psi}) \ldots f_{1}(\hat{\psi})\right](a, b)=\int d^{n} k d \mu[a, b, k] f_{N}\left(w\left(t_{N}\right)\right) \ldots f_{1}\left(w\left(t_{1}\right)\right) .
$$


Proof.

$$
\begin{aligned}
& \int d^{n} k d \mu[a, b, k] f_{N}\left(w\left(t_{N}\right)\right) \ldots f_{1}\left(w\left(t_{1}\right)\right) \\
& =\int d^{n} k d^{n N} \theta d^{n N} \rho\left\{\exp i\left(-\rho^{1} \cdot\left(\theta^{1}-b\right)-\cdots-\rho^{N} \cdot\left(\theta^{N}-\theta^{N-1}\right)-k \cdot\left(a-\theta^{N}\right)\right)\right. \\
& \left.f_{N}\left(\theta^{N}+i \rho^{N}\right) \ldots f_{1}\left(\theta_{1}+i \rho^{1}\right)\right\} \\
& \quad=\int d^{n} k d^{n N} \theta\left[f_{N}(\widehat{\psi})\right]\left(\theta^{N}, \theta^{N-1}\right) \ldots\left[f_{1}(\widehat{\psi})\right]\left(\theta^{1}, b\right) \delta\left(\theta^{N}, a\right)
\end{aligned}
$$

(using (2.18)),

$$
=\left[f_{N}(\hat{\psi}) \ldots f_{1}(\hat{\psi})\right](a, b) .
$$

(Equation (3.16) appears inconsistent because the right-hand side is manifestly $t_{i}$-dependent and the left-hand side not; in fact the $t$ dependence of the left-hand side is hidden in the ordering of the $t_{i}$.)

To end this section, an alternative version of Grassmann Brownian motion will be given, involving a Fourier expansion in terms of independent Gaussian variables. This version is useful when actually calculating fermionic path integrals, as will be clear from the example of the fermionic oscillator considered in the next section. This Fourier expansion corresponds to the physicist's technique of replacing the sum over paths by the sum over Fourier modes with anticommuting boundary conditions. As a preliminary the Gaussian process which it is natural to call the Grassmann Brownian bridge is required.

Definition 3.7. The $n$-dimensional Grassmann stochastic process $\{w(t)[0,0,0] \mid t \in$ $(0, \infty)\}$ is called the $n$-Grassmann Brownian bridge.

Two useful properties of this process are (a) that it is Gaussian, (as may be shown directly by calculating its distribution), and (b) that

$$
\begin{aligned}
& \operatorname{cov}\left(w^{i}(s), w^{j}(t)\right)=-i \delta^{i j} \quad \text { if } \quad s<t \\
& =0 \quad \text { if } \quad s=t \\
& =+i \delta^{i j} \quad \text { if } \quad s>t \text {. }
\end{aligned}
$$

The fact that Gaussian processes are uniquely characterised by their covariances will now be exploited in constructing the Fourier expanded version.

Proposition 3.8. Let $\left(X^{i r}, Y^{i r}\right), r=1,2, \ldots$ be independent $2 n$-dimensional Gaussian Grassmann random variables, each with distribution $d^{n} X d^{n} Y \exp -i Y \cdot X$, and let $\left(B_{L}^{(0, n) z},\left\{f_{N}\right\}, d \mu\right)$ be the corresponding probability measure space. Thus

$$
f_{N}\left(X^{i 1}, \ldots, X^{i N}, Y^{i 1}, \ldots, Y^{i N}\right)=\exp -i\left(\sum_{r=1}^{N} Y^{r} \cdot X^{r}\right) .
$$

Also let

$$
C_{r}=(2 r+1) \pi \quad(r=1,2, \ldots)
$$

and, for $s \in[0,1]$,

$$
Z_{N}^{i}(s)=\sum_{r=1}^{N}\left(2 / \sqrt{ } C_{r}\right)\left(\sin C_{r} s X^{i r}+i \cos C_{r} s Y^{i r}\right) .
$$

Then (i) the sequences $\left\{Z_{r}^{i}(s) \mid s \in[0,1]\right\}$ define an $n$-dimensional stochastic process $Z^{i}(s)$ on $\left(B_{L}^{(0, n) z},\left\{f_{N}\right\}, d \mu\right)$; (ii) the process is Gaussian; and 
(iii) covariance $\left(Z^{i}(s), Z^{j}(t)\right)=-i \delta^{i j}$ if $s<t$

$$
\begin{aligned}
& =0 \quad \text { if } s=t \\
& =i \delta^{i j} \quad \text { if } s>t \text {, }
\end{aligned}
$$

and hence $Z^{i}(t)$ is a version of the Grassmann Brownian bridge on $[0,1]$.

Proof. (i) Suppose that $v(1), \ldots, v(k)$ are in $M_{n}$ and $s_{1}, \ldots, s_{k}$ are in $[0,1]$. For $N$ an integer greater than zero, let

$$
h_{N}=\int d^{n N} X d^{n N} Y f_{N}(X, Y) Z_{N}^{v(1)}\left(s_{1}\right) \ldots Z_{N}^{v(k)}\left(s_{k}\right)
$$

Then

with

$$
h_{N+1}=h_{N}\left(1+a_{N}\right)
$$

$$
a_{N}=\sum_{P=1}^{n k} k_{N}^{P} C_{r}^{-p}
$$

where there exist constants $q^{u v}(u, v=1, \ldots, k)$ and $q^{p}(p=2, \ldots, n k)$ such that

and

$$
k_{N}^{1}=\sum_{u, v=1}^{k} q^{u v} \sin C_{r}\left(s_{u}-s_{v}\right)
$$

$$
\left|k_{N}^{P}\right|<q^{P}, \quad p=2, \ldots, n k .
$$

Hence $\sum a_{N}$ is convergent and $\sum\left(a_{N}\right)^{2}$ is absolutely convergent, and thus by standard theorems $h_{N}$ tends to a limit as $N$ tends to infinity.

(ii) and (iii). The sum of independent Gaussian Grassmann random variables of the same dimension is also a Gaussian Grassmann random variable, with covariance equal to the sum of the covariances of the independent random variables. Thus, for each $N=1,2, \ldots\left\{Z_{N}^{i}(s) \mid s \in[0,1]\right\}$ is a Gaussian process with

$$
\text { covariance }\left(Z_{N}^{i}(s), Z_{N}^{j}(t)\right)=\sum_{r=1}^{N} i \sin C_{r}(s-t) .
$$

Hence, since $Z^{i}(s)$ is the limit as $N$ tends to infinity of $Z_{N}^{i}(s)$, it is a Gaussian Grassmann stochastic process with

$$
\begin{aligned}
& \text { covariance }\left(Z^{i}(s), Z^{j}(s)\right)=\sum_{r=1}^{\infty} i \sin C_{r}(s-t) \\
& =\text { covariance }\left(w^{i}(s)[0,0,0], w_{j}(t)[0,0,0]\right)
\end{aligned}
$$

for $s, t$ in $[1,0]$ (using standard Fourier analysis).

Corollary 3.9. If $t>0 Z^{i}(s / t)+b^{i}+k^{i}$ is a version of $w^{i}(s)[b, b, k]$ on $(0, t)$.

\section{The Fermionic Feynman-Kac Formula}

Given a fermionic quantum mechanical system as described in Sect. 2, the time evolution equation (2.8) can be solved if one has an expression for the kernel of the evolution operator $e^{-H t}$. The theorem in this section (Theorem 4.2) establishes a Feynman-Kac formula, which expresses the kernel $\left[e^{-H t}\right](a, b)$ in terms of an 
integral with respect to anticommuting Wiener measure $\mu[a, b, k]$. First the random variable $\exp \left(-\int_{0}^{t} V(w(t)[a, b, k] d t)\right)$, which is the integrated in the Feynman-Kac formula, must be defined.

Proposition 4.1. Suppose that $V \in G H^{\infty}(n)$. Let

$$
C=[0, t]
$$

and, for $N=1,2, \ldots$, let

$$
B_{N}=\left\{0, t / 2^{N}, \ldots,\left(2^{N}-1\right) t / 2^{N}, t\right\}
$$

Also let random variables $g_{N}$ on $n$-Grassmann Wiener space with parameters $a, b, k$ be defined by

$$
g_{N}=\exp \left(-\sum_{r=1}^{2^{N}}\left(t / 2^{N}\right) V\left(w\left(r t / 2^{N}\right)\right)\right)
$$

Then the sequence $g_{N}$ defines a Grassmann random variable on n-Grassmann Wiener space. This random variable will be denoted $\exp \left(-\int_{0}^{t} V(w(t)[a, b, k] d t).\right)$

Proof. Let $f_{B}$ be the finite joint distributions of $n$-Grassmann Wiener measure with parameters $a, b, k$ (Eq. 3.15). Then, by Corollary 3.9, (and with the notation of Proposition 3.8)

$$
\begin{aligned}
\int & d^{n\left(2^{N}+1\right)} \theta d^{n\left(2^{N}+1\right)} \rho f_{B_{N}} g_{N} \\
= & \lim _{j \rightarrow \infty} \int d^{n k} X d^{n k} Y \exp \\
& \cdot\left(-i \sum_{r=1}^{j} X^{r} \cdot Y^{r}-\sum_{s=1}^{2^{N}}\left(t / 2^{N}\right) V\left(Z_{j}\left(s / 2^{N}\right)+b+k\right) \exp (-k \cdot(b-a))\right) \\
= & \left.\lim _{j \rightarrow \infty} h(N, j) \quad \text { (say }\right) .
\end{aligned}
$$

Now

$$
\begin{aligned}
& \lim _{N \rightarrow \infty} \sum_{s=1}^{2^{N}}\left(t / 2^{N}\right) V\left(Z_{j}\left(s / 2^{N}\right)+b+k\right) \\
& \quad=\int_{0}^{t} V\left(\sum_{r=1}^{j}\left(\sin C_{r} s X^{r}+\cos C_{r} s Y^{r}\right)+b+k\right) \\
& \quad=\sum_{\mu, v \in M_{n j}} P_{\mu v}(j) X^{v} Y^{v} \quad \text { (say). }
\end{aligned}
$$

Thus $h(N, j)$ tends to a limit $Q(j)$ as $N$ tends to infinity. Now

$$
Q(j)=\int d^{n j} X d^{n j} Y \exp -i \sum_{r=1}^{j} X^{r} \cdot Y^{r}-\sum_{\mu, v \in M_{n j}} P_{\mu v}(j) X^{\mu} Y^{v},
$$


and it can be shown that

$$
Q(j+1)=Q(j)\left(1+a_{j}\right)
$$

where $\sum a_{j}$ converges absolutely. Thus $\lim _{j \rightarrow \infty} \lim _{N \rightarrow \infty} h(N, j)$ exists and thus also $\lim \lim h(N, j)$ exists and so the sequence $g_{N}$ defines a Grassmann random variable. $N \rightarrow \infty j \rightarrow \infty$

Now the main theorem of this paper, which establishes the Feynman-Kac formula for fermions, can be given.

Theorem 4.2. Let $V$ be a function in $G H^{\infty}(n)$ with

and let

$$
V\left(\theta^{i}\right)=\sum_{\mu \in M_{n}} V_{\mu} \theta^{\mu}
$$

$$
H=V(\hat{\psi})
$$

(that is, $H=\sum_{\mu \in M_{n}} V_{\mu} \hat{\psi}^{\mu}$, with $\hat{\psi}^{\mu}$ the operator defined in Eq. (2.25)). Then, if $t>0$,

$$
\left[e^{-H t}\right](a, b)=\int d^{n} k d \mu[a, b, k] \exp \left(-\int_{0}^{t} V(w(t)) d t\right) .
$$

Proof.

$$
\begin{aligned}
& {[1-(t / m) V(\hat{\psi})](\theta, \phi)} \\
& \quad=\int d^{n} \rho d^{n} \theta \exp (-(t / m) V(\theta+i \rho)-i \rho \cdot(\theta-\phi))+o(1 / m) .
\end{aligned}
$$

Hence, by the compound interest formula,

$$
\left[e^{-t H}\right](a, b)=\lim _{m \rightarrow \infty} Q(m)(a, b),
$$

where

$$
\begin{aligned}
Q(m)(a, b)= & \int d^{n} k d^{n m} \theta d^{n m} \rho \exp \left\{\sum_{r=1}^{m}-(t / m) V\left(\theta^{r}+i \rho^{r}\right)-i\left(\rho^{1} \cdot\left(\theta^{1}-a\right)\right.\right. \\
& \left.\left.+\rho^{2}\left(\theta^{2}-\theta^{1}\right)+\cdots+\rho^{m} \cdot\left(\theta^{m}-\theta^{m-1}\right)+k \cdot\left(\theta^{m}-b\right)\right)\right\}
\end{aligned}
$$

Thus

$$
\begin{aligned}
{\left[e^{-H t}\right](a, b) } & =\lim _{N \rightarrow \infty} Q\left(2^{N}\right)(a, b) \\
& =\int d^{n} k d \mu[a, b, k] \exp -\int_{0}^{t} V(w(s)) d s .
\end{aligned}
$$

As a simple example of the use of this theorem, the kernel of the evolution operator of the two-dimensional fermionic oscillator will be calculated. This system is defined by the Hamiltonian

Hence

$$
H=i c \hat{\psi}^{1} \hat{\psi}^{2}
$$

$$
\left[e^{-H t}\right](a, b)=\int d^{n} k d \mu[a, b, k] \exp -\int_{0}^{t} i c w^{1}(s) w^{2}(s) d s .
$$


Thus, by Corollary 3.9 (which gives the Fourier expansion of $w(t)[b, b, k]$ ) and using the fact that

one finds that

$$
d \mu[a, b, k]=d \mu[b, b, k] \exp -i k \cdot(a-b),
$$

$$
\begin{aligned}
{\left[e^{-H t}\right](a, b)=} & \int d^{n} k \prod_{r=1}^{\infty} d^{2} X^{r} d^{2} Y^{r} \exp -i k \cdot(a-b) \\
& \cdot \exp \left\{\sum _ { r = 1 } ^ { \infty } \left[-i Y^{r} \cdot X^{r}-\left(2 i c t / C_{r}\right)\left(X^{1 r} X^{2 r}-Y^{1 r} Y^{2 r}\right)\right.\right. \\
& \left.-4 i c t C_{r}^{-3 / 2}\left(\left(b^{1}+k^{1}\right) X^{2 r}-\left(b^{2}+k^{2}\right) X^{1 r}\right)\right] \\
& \left.+\left(b^{1}+k^{1}\right)\left(b^{2}+k^{2}\right)\right\} .
\end{aligned}
$$

Using standard techniques of Grassmann Gaussian integration [9] gives

$$
\begin{aligned}
& {\left[e^{-H t}\right](a, b)} \\
& \quad=\int d^{n} k \exp -i k \cdot(a-b) \cosh c t \exp \left(-i\left(b^{1}+k^{1}\right)\left(b^{2}+k^{2}\right) \tanh c t\right) \\
& \quad=\int d^{n} k \exp -i k \cdot(a-b)\left\{\cosh c t-i\left(b^{1}+k^{1}\right)\left(b^{2}+k^{2}\right) \sinh c t\right\} .
\end{aligned}
$$

Obviously this result could be obtained in other ways; the purpose of developing a fermionic path integral is not to tackle the finite dimensional linear algebra which purely fermionic systems involve, but to extend analytic techniques used for bose systems to systems involving fermions and bosons, and in particular to supersymmetric systems.

The approach to fermionic path integration developed in this paper has deliberately been as closely parallel as possible to the bosonic method (as described by Simon [1]), so that the two methods can easily be combined when considering a system containing both bosons and fermions. The basic formalism described in this paper thus leads naturally to several possible developments - path integrals for supersymmetric systems, path integrals for fermions in curved space and path integration on supermanifolds---all of which are under active consideration by the author. First steps in a theory of supersymmetric path integration have recently been completed [3].

This paper demonstrates that Grassmann variable techniques are not simply formal tricks, but are capable of being incorporated into a rigorous analytic scheme. This should open up the possibility of new ways of handling fermionic fields, in addition to the Matthews-Salam approach [15], where fermions are integrated out at the outset.

\section{References}

1. Simon, B.: Functional integration and quantum mechanics. New York, San Francisco, London: Academic Press 1979

2. Bochner, S.: Stochastic processes. Ann. Math. 48, 1014-1061 (1947)

3. Rogers, A.: Supersymmetric path integration. Phys. Lett. B193, 48-54 (1987)

4. Alvarez-Gaumé, L.: Supersymmetry and the Atiyah-Singer index theorem. Commun. Math. Phys. 
90, 161-173, (1983); Friedan, D., Windey, P.: Supersymmetric derivation of the Atiyah-Singer index theorem and the chiral anomaly. Nucl. Phys. B235, 395-416 (1984)

5. Berezin, F. A., Marinov, M. S.: ZhETFPis. Red. 21, 678-180 (1975) [= JETP letters 21, 320-321 (1975)]

6. Dashen, R. F., Hasslacher, B., Neveu, A.: Semiclassical bound states in an asymptotically free theory. Phys. Rev. D12, 2443-2458, 1975

7. Fadeev, L. D., Slavnov, A. A.: Gauge fields, introduction to quantum theory. London, Amsterdam, Ontario, Sydney, Tokyo: Benjamin/Cummings 1980

8. It is not possible to give a full list; some examples are: Applebaum, D., Hudson, R. L.: Fermion Ito's formula and stochastic evolutions. Commun. Math. Phys. 96, 473 (1984); Barnett, C., Streater, R. F., Wilde, I. F.: The Ito-Clifford integral. J. Funct. Anal. 48, 172-212 (1982); Hudson, R., Lindsay, J. M.: A non-commutative martingale representation theorem for non-Fock quantum Brownian motion. J. Funct. Anal. 61, 202-221 (1985); Kummerer, B.: Markov dilations on W*-algebras. J. Funct. Anal. 63, 139 (1985); See also: Quantum probability and its applications. Accardi, L., von Waldenfels, W. (eds.). Lecture Notes in Mathematics, Vol. 1136. Berlin, Heidelberg, New York: Springer

9. Haba, Z.: Euclidean formulation of quantum spinning particles, Bielefeld preprint no 214, (1986)

10. DeWitt, B. S.: Supermanifolds. Cambridge, London, New York, New Rochelle, Melbourne, Sydney: Cambridge Univ. Press 1984.

11. Rogers, A.: A global theory of supermanifolds. J. Math. Phys. 21, 1352- 1365 (1980); Rogers, A.: Graded manifolds, supermanifolds and infinite-dimensional Grassmann algebras. Commun. Math. Phys. 105, 375-364 (1986)

12. Berezin, F. A.: The method of second quantization. New York: Academic Press 1966

13. Seiler, E.: Constructive quantum field theory: Fermions. In: Gauge theories: Fundamental Interactions and rigorous results. Dita, P., Georgescu, V., Purice, R. (eds.). Boston, Basel, Stuttgart: Birkhäuser 1982 (and references therein); Kupsch, J.: Measures for fermionic integration. Universität Kaiserslautern preprint, April 1986

14. Segal, I. E.: A non-commutative extension of abstract integration. Ann. Math. 58, 595-596 (1953)

15. Matthews, P. T., Salam, Abdus: Propagators of quantised fields. Nuovo Cimento 2, 120-134 (1955); Gross, L.: On the formula of Matthews and Salam. J. Funct. Anal. 25, 162-209 (1977)

Communicated by B. Simon

Received December 22, 1986; in revised form March 30, 1987 